\title{
A NOTE ON COMPLEMENTARITY PROBLEM
}

\author{
ANTONIO CARBONE \\ Università degli Studi della Calabria \\ Dipartimento di Matematica \\ I-87036 Arcavacata di Rende (Cosenza), ITALY
}

(Received November 12, 1996)

\begin{abstract}
In this paper we prove a result of complementarity problem where compact condition is somewhat relaxed.
\end{abstract}

KEY WORDS AND PHRASES: Complementarity problem, variational inequality, implicit complementarity problem

1991 AMS SUBJECT CLASSIFICATION CODES: Primary 47H10, Secondary 54H25

Recently several interesting results have been given for complementarity problems As the complementarity problem, variational inequality and fixed point theory are closely related (equivalent to each other) that is why it has growing interest and varied applications. The applications in the field of economics, optimization, game theory, mechanics and engineering are even growing rapidly

Here we will start with implicit complementarity problem and then derive results for complementarity theory. For terminology one is referred to [1]

Let $\left\langle E, E^{*}\right\rangle$ be a dual system of a locally convex space. Let $K \subset E$ be a closed convex cone We denote by $K^{*}$ the dual cone of $K$, that is

$$
K^{*}=\left\{y \in E^{*}:\langle x, y\rangle \geq 0 \text { for all } x \in K\right\} .
$$

Let $f: K \rightarrow E^{*}$ and $g: K \rightarrow K$. Then the implicit complementarity problem is as follows

Find an $x_{0} \in K$ such that $g x_{0} \in K, f x_{0} \in K^{*}$ and

$$
\left\langle g x_{0}, f x_{0}\right\rangle=0 \ldots \text {. }
$$

The corresponding variational inequality will be as given below.

( $\beta$ ) Find $x_{0} \in K$ such that $g x_{0} \in K$ and $\left\langle x-g x_{0}, f x_{0}\right\rangle \geq 0$ for all $x \in K$

In [1] it is shown that $(\alpha)$ and $(\beta)$ are equivalent.

Indeed, if $(\beta)$ holds then by taking $g x_{0}=0$, we get $\left\langle x, f x_{0}\right\rangle \geq 0$ for all $x \in K$, so $f x_{0} \in K^{*}$

Also, if $x=0$ we get $\left\langle g x_{0}, f x_{0}\right\rangle \leq 0$ and if $x=2 g x_{0}$, then $\left\langle g x_{0}, f x_{0}\right\rangle \geq 0$.

Thus $\left\langle g x_{0}, f x_{0}\right\rangle=0$ and $(\alpha)$ is obtained.

In case $(\alpha)$ holds then $g x_{0} \in K, f x_{0} \in K^{*}$ and $\left\langle g x_{0}, f x_{0}\right\rangle=0$.

Since $f x_{0} \in K^{*}$ so $\left\langle x, f x_{0}\right\rangle \geq 0$ for all $x \in K$.

Hence $\left\langle x-g x_{0}, f x_{0}\right\rangle \geq 0$ for all $x \in K$ and $(\beta)$ holds.

The following result given in [2] extends and unifies results due to park and Kim [3], Takahashi [4] and Chitra and Subrahmanyan [5]. The proof in [2] is based on the KKM-map principle (for details see Granas [6]).

THEOREM 1. Let $C$ be a nonempty convex subset of a topological vector space $X$. Let $A \subset C \times C$ and $g: C \rightarrow C$ such that the following conditions are satisfied. 
i) $(x, g x) \in A$ for all $x \in C$;

ii) for each $y \in C$, the set $\{x \in C:(x, g y) \notin A\}$ is convex or empty,

iii) for each $x \in C$, the set $\{y \in C:(x, g y) \in A\}$ is closed in $C$;

iv) $C$ has a nonempty compact convex subset $C_{0}$ such that the set $D=\{y \in C:(x, g y) \in A$ for all $x \in C_{0}$ \} is compact

Then there exists a $y_{0} \in C$ such that $C \times\left\{g y_{0}\right\} \subset A$.

NOTE. If $g=I$, an identity function, then one gets that $C \times\left\{y_{0}\right\} \subset A$ [7].

In case $C$ is compact convex and $g$ is an identity map then we have the following

COROLLARY 1. Let $C$ be a nonempty compact convex subset of a topological vector space $X$ and let $A \subset C \times C$ have the following:

i) $(x, x) \in A$ for each $x \in C$,

ii) for each $y \in C$ the set $\{x \in C:(x, y) \notin A\}$ is convex or empty;

iii) for each $x \in C$ the set $\{y \in C:(x, y) \in A\}$ is closed in $C$.

Then there is $y_{0} \in C$ such that $C \times\left\{y_{0}\right\} \subset A$.

We will make use of Theorem 1 to prove the following result in complementary problem. First we give our result for variational inequality and then as a consequence derive complementarity problem

THEOREM 2. Let $D$ be a nonempty convex subset of a topological vector space $E$ If $f: D \rightarrow E^{*}$ and $g: D \rightarrow D$ are continuous functions such that

$$
\langle g x, f x\rangle \leq\langle x, f x\rangle \text { for all } x \in D .
$$

Let $D$ have a nonempty compact convex subset $D_{0}$ such that the set

$$
B=\left\{y \in D:\langle g y, f y\rangle \geq 0 \text { for all } x \in D_{0}\right\}
$$

is compact

Then there is $y_{0} \in D$ such that

$$
\left\langle x-g y_{0}, f y_{0}\right\rangle \geq 0 \text { for all } x \in D .
$$

PROOF. Let $A=\{(x, y) \in D \times D:\langle x-g y, f y\rangle \geq 0\}$. Then $(x, x) \in A$ by hypothesis

Since $f$ and $g$ are continuous and the bilinear form $\langle$,$\rangle is continuous therefore the set$

$$
\{y \in D:\langle x-g y, f y\rangle \geq 0\}
$$

is closed for every $x \in D$

The set

$$
A_{x}=\{x \in D:(x, y) \notin A\}=\{x \in D:\langle x-g y, f y\rangle<0\}
$$

is convex.

Indeed, if $x_{1}$ and $x_{2}$ are in $A_{x}$ then $z=\lambda x_{1}+(1-\lambda) x_{2} \in A_{x}$, where $0 \leq \lambda \leq 1$.

We write

$$
\begin{aligned}
\langle z-g y, f y\rangle & =\left\langle\lambda x_{1}+(1-\lambda) x_{2}-g y, f y\right\rangle \\
& =\left\langle\lambda x_{1}-g y, f y\right\rangle+\left\langle(1-\lambda) x_{2}-g y, f y\right\rangle \\
& =\lambda\left\langle x_{1}-g y, f y\right\rangle+(1-\lambda)\left\langle x_{2}-g y, f y\right\rangle \\
& <\lambda .0+(1-\lambda) \cdot 0=0 .
\end{aligned}
$$

So $\langle z-g y, f y\rangle<0$; and $A_{x}$ is convex.

Thus conditions of Theorem 1 are satisfied and there is a $y_{0} \in D$ such that

$$
\left\langle x-g y_{0}, f y_{0}\right\rangle \geq 0 \text { for all } x \in D .
$$

Noting that the variational inequality is equivalent to the complementarity problem we derive the following 
Under the hypotheses of Theorem 2 we get that there is a $y_{0} \in D$ such that $g y_{0} \in D, f y_{0} \in D^{*}$ and $\left\langle g y_{0}, f y_{0}\right\rangle=0$

In case $g=I$, an identity function, then we get a complementarity problem, that is, there is a $y_{0} \in D$ such that $f y_{0} \in D^{*}$ and $\left\langle y_{0}, f y_{0}\right\rangle=0$.

In case $D$ is also a compact set then the following result is obtained as a corollary This is due to Isac [8].

COROLLARY 2. Let $D$ be a nonempty compact convex subset of $E$ and $f: D \rightarrow E^{*}, g: D \rightarrow D$ continuous maps. If $\langle x-g x, f x\rangle \geq 0$ for all $x \in D$, then there is a $y_{0} \in D$ such that

$$
\left\langle x-g y_{0}, f y_{0}\right\rangle \geq 0 \text { for all } x \in D .
$$

We note that $E$ need not be a Hausdorff space.

In case $\left\langle E, E^{*}\right\rangle$ is a dual system of Banach space $E, K$ is a closed convex cone in $E$ and $D$ is a nonempty compact convex subset of $K$, then we obtain the following

THEOREM 3. Let $f, g: K \rightarrow E^{*}$ satisfy the following:

i) $\langle g x, f x\rangle \geq\langle x, f x\rangle$ for every $x \in D$;

ii) for each sequence $\left\{y_{n}\right\}$ in $D$ weakly converging to $y_{0}$, then

$$
\liminf \left\langle x, f x_{n}\right\rangle \leq\left\langle x, f y_{0}\right\rangle \text { for every } x \in D \text {; }
$$

iii) $x \rightarrow\langle g x, f x\rangle$ is sequentially weakly lower semicontinuous on $D$. Then there is an $x_{0} \in D$ such that

$$
\left\langle x-g x_{0}, f x_{0}\right\rangle \geq 0 \text { for all } x \in D .
$$

In the proof of this result one can use Corollary 1 in the setting of weak topology.

PROOF. Let $a=\{(x, y) \in D \times D:\langle g y-x, f y\rangle \leq 0\}$.

Then, on the same lines as given in [1] one gets that

i) $(x, x) \in A$ for every $x \in D$;

ii) the set $\{x \in D:(x, y) \notin A\}$ is convex for every $y \in D$;

iii) the set $\{y \in D:(x, y) \in D\}$ is weakly closed.

Hence the result follows from Corollary 1 in the weak topology sense.

\section{REFERENCES}

[1] ISAC, G., Complementarity Problems, Springer-Verlag, New York, 1992.

[2] CARBONE, A., Variational inequalities and fixed points, Indian J. Math., 36 (1994), 193-200

[3] PARK, S. and KIM, I., Nonlinear variational inequalities and fixed point theorems, Bull. Korean Math. Soc., 26 (1989), 139-149.

[4] TAKAHASHI, W., Nonlinear variational inequalities and fixed point theorems, J. Math. Soc. of Japan, 28 (1976), 168-181.

[5] CHITRA, A. and SUBRAHMANYAM, P.V., A generalization of a section theorem of Ky Fan and its applications to variational inequalities, Review of Research, University of Novi Sad, Math. Series, 17 (1987), 17-37.

[6] GRANAS, A., KKM-maps and their applications to nonlinear problems, The Scottish Book, Ed R.D. Mauldin, Birkhauser (1982), 45-61.

[7] LIN, T.C., Convex sets, fixed points, variational and minimax inequalities, Bull. Austr. Math. Soc. 34 (1986), 107-117.

[8] ISAC, G., A special variational inequality and the implicity complementarity problem, J. Fac. Sci., Univ. of Tokyo, 37 (1990), 109-127. 


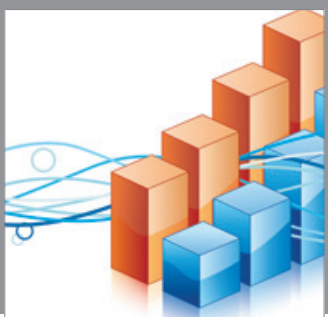

Advances in

Operations Research

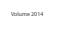

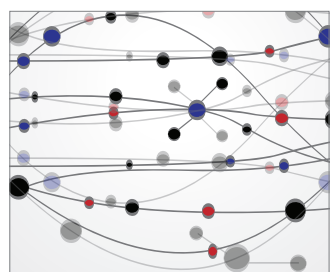

\section{The Scientific} World Journal
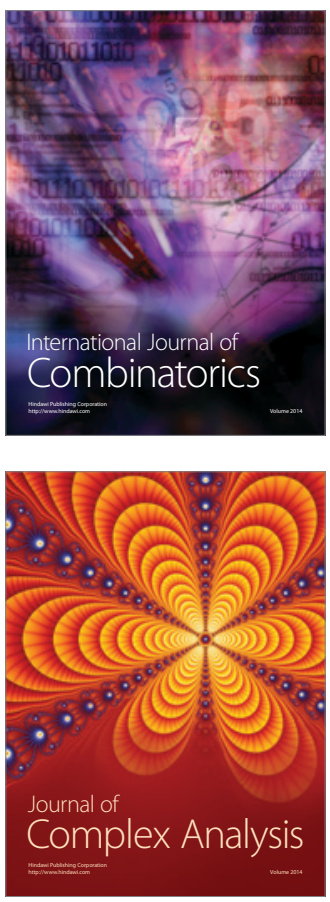

International Journal of

Mathematics and

Mathematical

Sciences
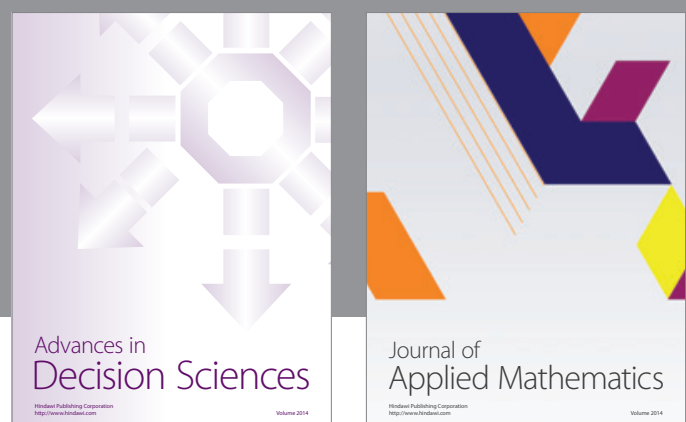

Journal of

Applied Mathematics
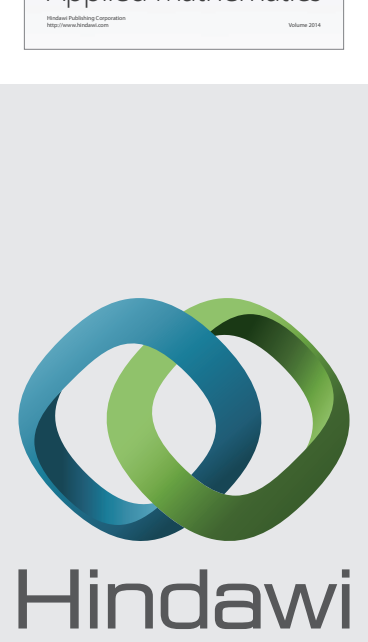

Submit your manuscripts at http://www.hindawi.com
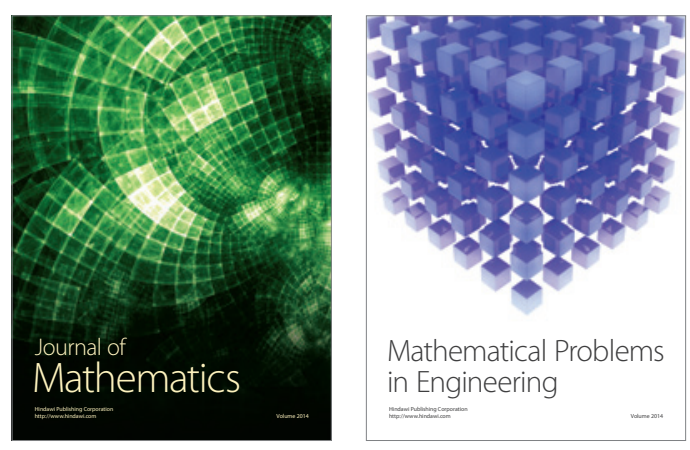

Mathematical Problems in Engineering
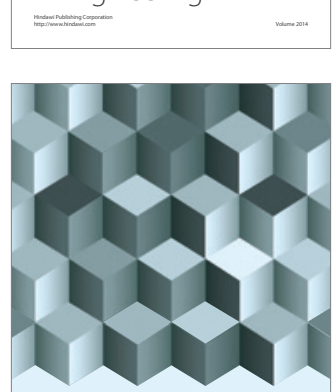

Journal of

Function Spaces
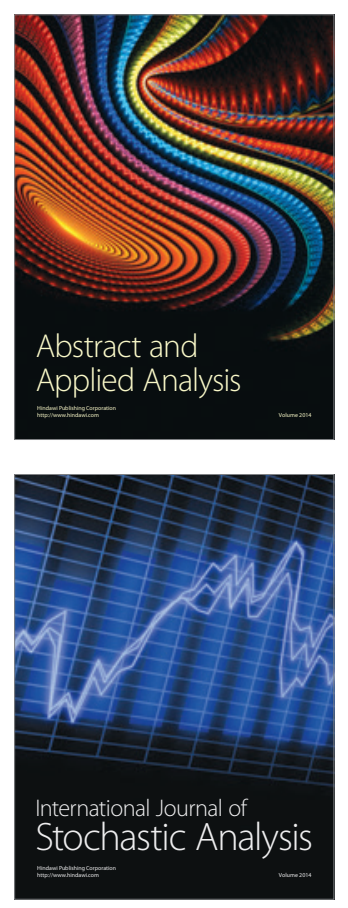

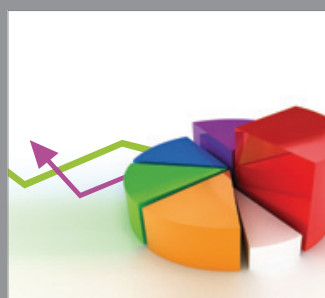

ournal of

Probability and Statistics

Promensencen
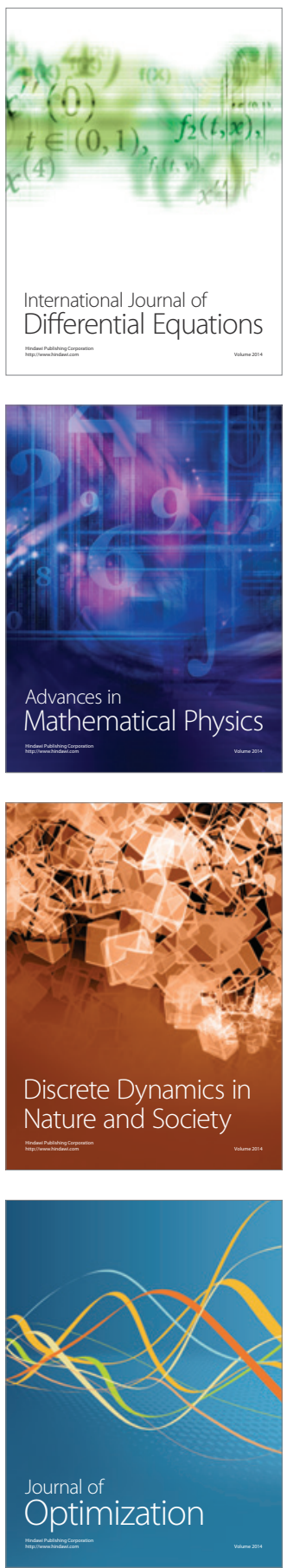